\title{
Journey into the unknown: a survey into the effects of ageing on the health of people with spina bifida and/or hydrocephalus in the West Midlands area

\author{
Geraldine Long* and Jenny Green*
}

Address: ASBAH House, 42 Park Road, Peterborough PE1 2UQ, UK

Email: Geraldine Long* - geraldinel@ashbah.org; Jenny Green* - jennyg@asbah.org

* Corresponding authors

from 53rd Annual Meeting of the Society for Research into Hydrocephalus and Spina Bifida Belfast, UK. 24-27 June 2009

Published: 27 November 2009

Cerebrospinal Fluid Research 2009, 6(Suppl 2):S29 doi:10.1186/1743-8454-6-S2-S29

This abstract is available from: http://www.cerebrospinalfluidresearch.com/content/6/S2/S29

(c) 2009 Long and Green; licensee BioMed Central Ltd.

\section{Background}

This study looked at the effects of ageing on the health of 42 adults with spina bifida (SB) and/or hydrocephalus $(\mathrm{H})$ over the age of 35 years, living in the West Midlands Region of the United Kingdom, and known to the authors, who are employed as Advisers with ASBAH. Of the 14 males and 28 females who took part, 9 had SB, 27 SB\&H, $4 \mathrm{H}$ and 2 spina bifida occulta. The focus of the project was identified in preliminary exploration with a group of service users and emphasis given to general health, mobility, continence and hydrocephalus.

\section{Materials and methods}

An initial simple questionnaire about health issues was sent to 180 people of whom 42 volunteered to take part in a more detailed study. Confidentiality was assured. A questionnaire was devised and the interviews conducted by the authors. The results were collated into a database for study. At the request of the focus group an attempt was made to identify feelings about the effects of ageing.

\section{Results}

Participants revealed widespread concern related to general health; for example 26/42 had experienced skin problems, 31/42 were concerned about weight and 17/42 had high blood pressure. Deteriorating eyesight and memory were reported by many. Depression was a significant feature in over half of the respondents. The psychological and physical impact of deteriorating mobility was a key finding; 18 were wheelchair users as children but this figure had risen to 26 in adulthood. A review of bladder and bowel continence management showed little evidence of access to modern methods. Shunting remained the main treatment of hydrocephalus but a few revisions were reported. Tables and charts illustrating the results, and quotes from participants are included in the text.

\section{Conclusion}

The project provided a snapshot of the health challenges faced by 42 adults living with SB and/or $\mathrm{H}$ and offered an opportunity for them to have a voice, to inform debate among themselves, carers and professionals, in order to make a positive contribution to the understanding of their condition during the ageing process. It also provoked ideas for further research into the world of living with this complex condition; e.g. exploring the capacity of those with SB/H to consider change in continence management, or an assessment of the impact of adopting wheelchair use during adulthood. Comparative studies with other age groups i.e. 18-35 yrs and 10-18 yrs might also provide a valuable insight into how changing treatments and attitudes might result in different outcomes for today's young people with SB and/or $\mathrm{H}$. 